\title{
Checklist of ectoparasitic arthropods among cave-dwelling bats from Marinduque Island, Philippines
}

\author{
Ace Kevin S. Amarga ${ }^{1,4}$, Phillip A. Alviola ${ }^{2,3}$, Ireneo L. Lit, Jr. ${ }^{2,3}$ \& Sheryl A. Yap ${ }^{1,2}$ \\ ${ }^{1}$ Crop Protection Cluster, College of Agriculture, University of the Philippines Los Baños, Laguna 4031, Philippines \\ ${ }^{2}$ Museum of Natural History, University of the Philippines Los Baños, College, Laguna 4031, Philippines \\ ${ }^{3}$ Institute of Biological Sciences, University of the Philippines Los Baños, Laguna 4031, Philippines \\ ${ }^{4}$ Corresponding author. E-mail: ace_amarga061@yahoo.com
}

\begin{abstract}
This paper constitutes the first ectoparasite faunal survey of bats for Marinduque Island, Philippines. From 1-12 June 2010, 150 bats belonging to 11 species were captured in 11 caves on the island. Each bat was sampled for ectoparasitic arthropods, and a total of 587 individuals representing 21 species, belonging to five families (Acari: Argasidae and Spinturnicidae; Diptera: Nycteribiidae and Streblidae; and Siphonaptera: Ischnopsyllidae) were collected. New host records (new host record) in the Philippines for Brachytarsina cucullata Jobling 1934, B. proxima Jobling 1951, B. werneri Jobling 1951, Raymondia pseudopagodarum Jobling 1951, Eucampsipoda philippinensis Ferris 1924, Nycteribia allotopa Speiser 1901, Nycteribia allotopoides Theodor 1963, Nycteribia parvuloides Theodor 1963, Ancystropus taprobanius (Turk 1950), and Carios batuensis Hirst 1929 were documented. A checklist of the ectoparasitic species known from the Philippines, their distribution, and bat host species is provided.
\end{abstract}

Key words: Acari; batflies; Diptera; fleas; Streblidae; mites

\section{INTRODUCTION}

Bat ectoparasites constitute a specialized guild of species that have evolved a plethora of morpho-physiological adaptations including usually dorsoventrally flattened bodies, reduced eyesight, and presence of ctenidia and/or setae. They thrive on the wing membranes and bodies of their respective hosts where they feed on blood. There are six insect families known to be ectoparasitic on chiropterans and five of these are exclusively parasitize bats; they are the Arixeniidae (Dermaptera, "bat earwigs") (NAKATA \& MAA 1974), Polyctenidae (Hemiptera: Heteroptera, "bat bugs"), Nycteribiidae and Streblidae (Diptera, "bat flies"), and Ischnopsyllidae (Siphonaptera, "bat fleas") (MARSHALL 1982). Although the family Cimicidae is not exclusively ectoparasitic on bats, it contains species such as Cimex pilosellus (Horvath, 1910) and C. pipistrelli Jenyns, 1839 which are ectoparasitic on bats. Mites and ticks (Acari) are also documented to parasitize bats, including the families Chirodiscidae (FAIN 1980), Chirorhynchobiidae (BосHKOV et al. 2008), Sarcoptidae (KLOMPEN \& O'CONNOR 1987), Ereynetidae (FAIN \& Lukoschus 1971), Laelapidae (SHAW 2011), Macronyssidae (TIPTON \& BoESE 1958; HedDERgOTT 2008), Myobiidae (FAIN 1979; UchiKaWA 1985; EstradAPeña And De La Cruz 1992), Rosensteiniidae (Durden et al. 1992), Spinturnicidae (Cuy 1980a, 1980b; Delfinado \& BAKER 1963, BAKer \& Delfinado 1964), and Argasidae (DURDEN et al. 2008). Studies on the taxonomy of bat ectoparasites in the Philippine Islands have been few, and among them are those conducted by JORDAN \& Rothschild (1921), TheOdor (1963), DelFinado \& BAKER (1963), Cuy (1980a, 1980b), FAIN (2002), Hastriter (2007), ZABAT \& EDUARDO (2011), HASTRITER \& BUSH (2013), and Alvarez et al. (2015). Throughout the history of bat ectoparasite taxonomy research in the Philippines, Marinduque Island has hitherto remained unstudied. This study aims to document the ectoparasite fauna of cavedwelling chiropterans in Marinduque Island, especially because of the increasing rate of human disturbance including tourist visits to caves in that province.

\section{MATERIALS AND METHODS}

\section{Study site}

Bats were captured using $3 \times 2.5 \mathrm{~m}$ mobile mist nets from 2-11 June 2010. Collections were made in different chambers and along trails in caves at varying elevations on Marinduque Island. Caves were in the municipalities of Boac, Mogpog, Sta. Cruz, and Torrijos (Figure 1; Table 1).

\section{Data collection}

Collected host species were identified up to species level. Some specimens were euthanized as voucher specimens by placing them inside $\mathrm{ZipLoc}^{\mathrm{TM}}$ resealable plastic bags with cotton impregnated with ethyl acetate while others were 


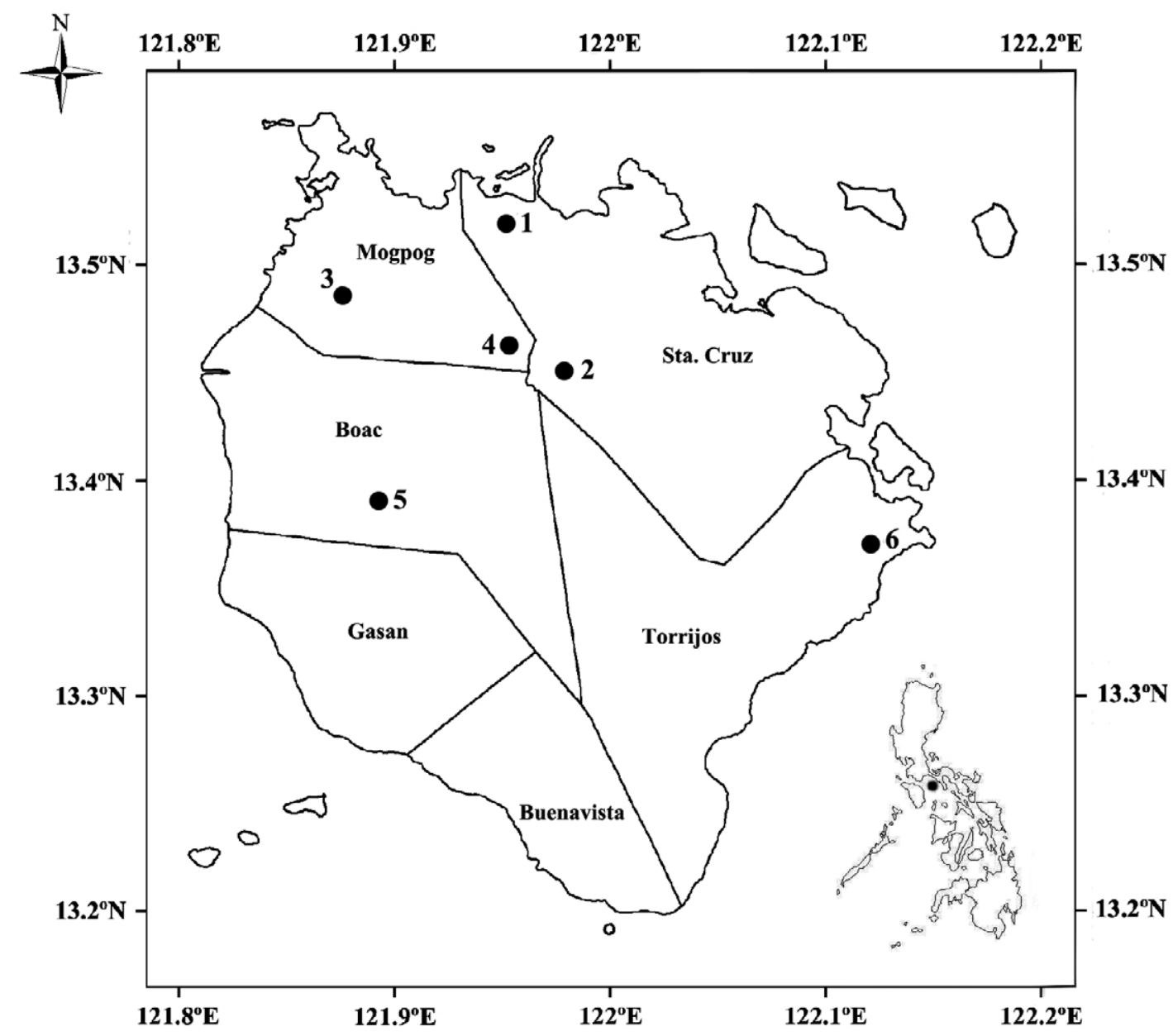

Figure 1. Sampling Sampling sites. 1: Bagongbongan Cave, Barangay Isidro, 2: Lamesa Cave, Barangay Lamesa. 3: Buaya Cave, Barangay Bintakay. 4: Tarug 3+4 cave, Barangay Tarug. 5: Bungoi Cave, Talao 1 and 2 caves, Barangay Duyay. 6: Ka Amundos Cave, Barangay Bonliw.

Table 1. Sampling sites with coordinates and elevation.

\begin{tabular}{llccc}
\hline Cave & Location & Latitude & Longitude & Elevation (m) \\
\hline Bagongbongan & Barangay Isidro, Sta. Cruz & $13^{\circ} 50^{\prime} 56.6^{\prime \prime} \mathrm{N}$ & $121^{\circ} 57^{\prime} 04.3^{\prime \prime} \mathrm{E}$ & \\
Lamesa & Barangay Lamesa, Sta. Cruz & $13^{\circ} 29^{\prime} 32.1^{\prime \prime} \mathrm{N}$ & $121^{\circ} 57^{\prime} 05.6^{\prime \prime} \mathrm{E}$ & 206 \\
Tarug (3+4) & Barangay Tarug, Mogpog & $13^{\circ} 30^{\prime} 03.0^{\prime \prime} \mathrm{N}$ & $121^{\circ} 56^{\prime} 16.9^{\prime \prime} \mathrm{E}$ & 271 \\
Buaya & Barangay Bintakay, Mogpog & $13^{\circ} 29^{\prime} 29.3^{\prime \prime} \mathrm{N}$ & $121^{\circ} 52^{\prime} 33.6^{\prime \prime} \mathrm{E}$ & 152 \\
Bungoi & Barangay Duyay, Boac & $13^{\circ} 21^{\prime} 34.7^{\prime \prime} \mathrm{N}$ & $121^{\circ} 53^{\prime} 29.7^{\prime \prime} \mathrm{E}$ & 357 \\
Talao 1 & Barangay Duyay, Boac & $13^{\circ} 21^{\prime} 31.1^{\prime \prime} \mathrm{N}$ & $121^{\circ} 53^{\prime} 31.6^{\prime \prime} \mathrm{E}$ & 414 \\
Talao 2 & Barangay Duyay, Boac & $13^{\circ} 21^{\prime} 28.9^{\prime \prime} \mathrm{N}$ & $121^{\circ} 53^{\prime} 32.9^{\prime \prime} \mathrm{E}$ & 438 \\
Ka Amundos & Barangay Bonliw, Torrijos & $13^{\circ} 21^{\prime} 59.6^{\prime \prime} \mathrm{N}$ & $121^{\circ} 06^{\prime} 46.6^{\prime \prime} \mathrm{E}$ & 76 \\
\hline
\end{tabular}

released. The entire dermal surface of the host was examined systematically for the presence of ectoparasites with particular attention to parasitic arthropods, especially on the wing and tail membranes, ventral and dorsal parts of the ears and pelage, toes and wing ridges. Fine-pointed forceps were used to remove the ectoparasites from host animals.

After removal from their hosts, the ectoparasites were counted and specimens placed in 95\% ethanol in Eppendorf $^{\mathrm{TM}}$ vials that were labeled with place and date of collection as well as host data. To avoid repetitious mention of general collection data in the citations of material examined, all ectoparasites were collected by A.K.S. Amarga and P.A. Alviola in June 2010 from Marinduque Island.
Identifications of ectoparasites were made using a stereomicroscope. Available taxonomic keys to various ectoparasitic taxa infesting bats were used to identify the ectoparasites to the lowest taxonomic level. Voucher specimens of both hosts and ectoparasites were deposited in the University of the Philippines Los Baños, Museum of Natural History (UPLB MNH).

\section{RESULTS}

A total of 587 insect and acarine ectoparasites, belonging to five families, were obtained from 150 individuals of cave bats representing 11 species (Table 2). New island and host bat records are also reported. 


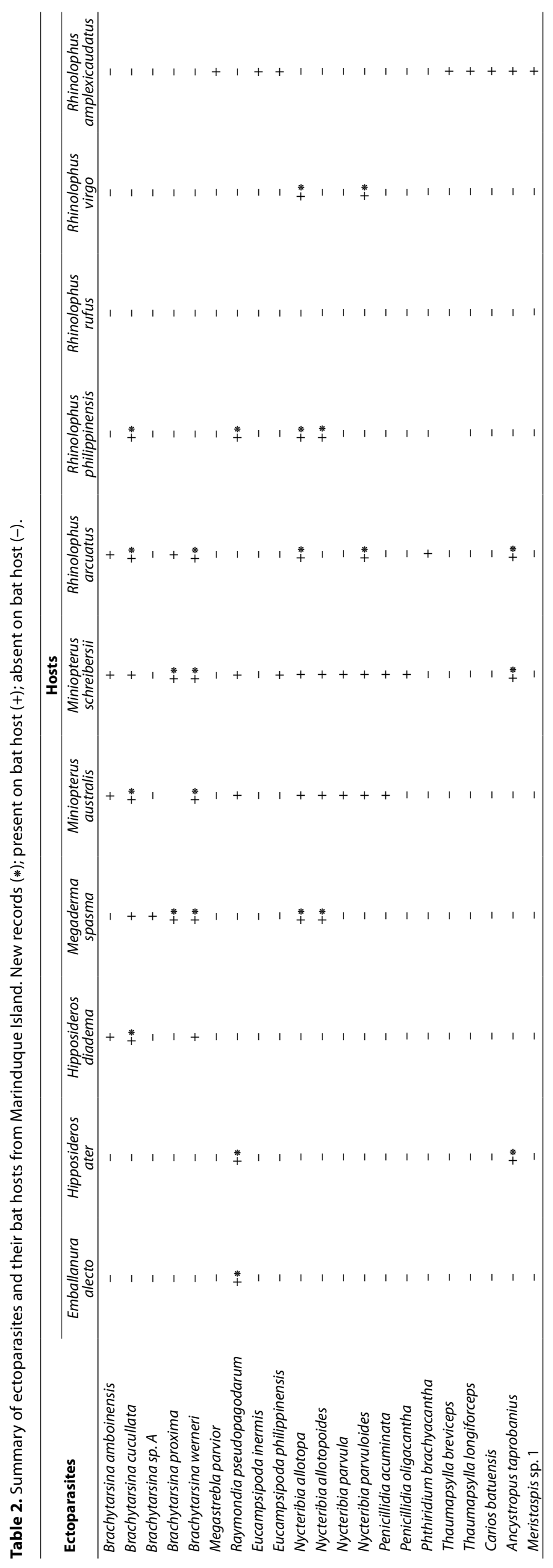

Class Insecta

Subclass Pterygota

Order Diptera

Suborder Brachycera

Division Cyclorrhapha

Superfamily Hippoboscoidea

Family Streblidae Kolenati 1863

Subfamily Brachytarsininae Speiser 1900

Genus Brachytarsina Macquart 1851

Brachytarsina MACQUART (1851): 280.

Nycteribosca SPEISER (1900): 48.

Type species: Brachytarsina flavipennis Macquart 1851.

Brachytarsina amboinensis Rondani, 1878

Brachytarsina amboinensis RoNDANI (1878): 166. Type: host unknown, Amboina, Moluccas; MAA (1965): 383; Delfinado \& HARdy (1977): 433.

Nycteribosca amboinensis SPEISER (1900): 31.

Documented host records: Eonycteris spelaea, Hipposideros coronatus, Miniopterus australis, Miniopterus schreibersi, Miniopterus tristis, Rhinolophus spp., and Rousettus amplexicaudatus (Cuy 1980a).

Material examined: on Hipposideros diadema: $4 \hat{\jmath}$,,+ ; on M. australis: $\hat{\sigma}$, + ; on M. schreibersi: $3 \hat{\jmath}$,,+ ; on Rhinolophus arcuatus: $\hat{\jmath}$.

Distribution: Australia; Malaysia (Indonesia (Amboin Island), Myanmar, Philippines (Greater Luzon: Marinduque Island (new island record), Polillo Islands, Tablas Island; Greater Mindanao); Japan, Taiwan.

Brachytarsina cucullata (Jobling, 1934)

Nycteribosca cucullata Jobling (1934): 71. Type: male, on Taphozous melanopogon Temminck, Matugama state, Kalutara, Ceylon, in Natural History Museum (London); JOBLING (1951): 230; MAA (1962): 433.

Brachytarsina cucullata MAA (1965): 383; DELFINAO \& HARDY (1977): 433.

Documented host records: Cynopterus brachyotis, Hipposideros diadema (new host record), Megaderma spasma (new host record), Miniopterus australis (new host record), Miniopterus schreibersi, Rhinolophus arcuatus (new host record), Rhinolophus philippinensis (new host record), Taphozous philippinensis.

Material examined: on $H$. diadema: $2 \uparrow$; on $M$. spasma: 2q; on M. australis: $2 \hat{\jmath}$; on $M$. schreibersi: $7 \hat{\delta}, 3 \bigcirc$; on $R$. arcuatus: $\widehat{\delta}$; on $R$. philippinensis: $\widehat{\delta}$.

Distribution: Malaysia, Philippines (Greater Luzon: Marinduque Island (new island record); Greater Mindanao); Sri Lanka.

\section{Brachytarsina sp. A}

Brachytarsina sp. A CuY (1980a): 140

Documented host record: Megaderma spasma.

Material examined: on M. spasma: $3 \hat{\jmath}, 4$ ㅇ․

Distribution: Philippines (Greater Luzon: Bataan, Laguna, Marinduque Island (new island record). 


\section{Brachytarsina proxima (Jobling, 1951)}

Nycteribosca proxima Jobling (1951): 238. Type: on Rousettus amplexicaudatus (Geoffroy), Luangbay Cave, Sitio Tegato, Davao City, Mindanao, Philippines; in Field Museum of Natural History (Chicago).

Brachytarsina proxima MAA (1965): 383; DELFINADO \& HARDY (1977): 433.

Documented host records: Eonycteris spelaea, Hipposideros diadema, Megaderma spasma (new host record), Miniopterus schreibersi (new host record), Rhinolophus arcuatus, Rhinolophus rufus, Rousettus amplexicaudatus.

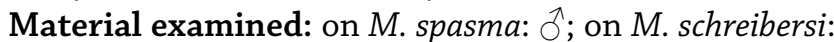
$4 \hat{O}$,,+ ; on $R$. arcuatus: $2 \hat{\jmath}$.

Distribution: Philippines (Greater Luzon: Marinduque (new island record); Greater Mindanao; Greater Palawan).

\section{Brachytarsina werneri (Jobling, 1951)}

Nycteribosca werneri Jobling (1951): 238. Type: on Hipposideros diadema griseus (Meyen), Central Cave, Cotabato City, Mindanao, Philippines, in Field Museum of Natural History (Chicago); MAA (1962): 434

Brachytarsina werneri MAA (1965): 383; DELFINADO \& HARDY (1977): 434.

Documented host records: Eonycteris robusta, Hipposideros diadema, Megaderma spasma (new host record), Miniopterus australis (new host record), $M$. schreibersi (new host record), Rhinolophus arcuatus (new host record).

Material examined: on $H$. diadema: $2 \hat{\sigma}, 3+$; on $M$. spasma: $2 \hat{O}$; on $M$. australis: $\hat{\sigma}$; on $M$. schreibersi: $4 \hat{O}, 6$; ; on R. arcuatus: $3 \hat{\jmath}$.

Distribution: Philippines (Greater Luzon: Marinduque Island (new island record); Greater Mindanao).

Genus Megastrebla Maa, 1971

Megastrebla MAA (1971): 230. Type species: Nycteribosca nigriceps Jobling (1934), by original designation. DelfinAdo \& HARDY (1977): 434

Megastrebla parvior (Maa, 1962)

Nycteribosca gigantea SPEISER (1900): 31-70. Type: on Dobsonia peroni (Geoffroy), Bismarck Archipelago, in Natural History Museum Giacomo Doria (Genoa); FerRIS (1924): 73, pro parte; JoBLING (1951): 230

Nycteribosca parvior MAA (1962): 433. Type: on Eocnycteris spelaea Dobson, Batu Caves, Selangor, Malaysia, in Bernice Pauahi Bishop Museum (Honolulu).

Brachytarsina parvior MAA (1965): 383.

Megastrebla parvior MAA (1971): 222; DELFINADO \& HARDY (1977): 434.

Documented host records: Eonycteris robusta, E. spelaea, Hipposideros diadema, Miniopterus australis, M. schreibersi, Rousettus amplexicaudatus.

Material examined: on Rousettus amplexicaudatus: $4 \hat{} \hat{\gamma}, 5$ ㅇ. Distribution: India, Indonesia (Borneo, Java, Sumatra, Sumba), Malaysia, Myanmar, Philippines (Greater Luzon: Marinduque Island, new island record; Polillo Islands; Greater Mindanao: Leyte, Samal; Greater Negros-Panay: Negros, Cebu; Greater Palawan: Palawan mainland, Balabac Island).
Genus Raymondia Frauenfeld, 1856

Raymondia FraUenfeld (1856): 323-328. Type: Raymondia huberi 1855, by subsequent designation of SPEISER (1900): 49; JOBLING (1930): 283; JoBLING (1951): 228; MAA (1962): 435; MAA (1965): 384; DELFINADO \& HARDY (1977): 434.

\section{Raymondia pseudopagodarum Jobling, 1951}

Raymondia pseudopagodarum JoBling (1951): 241. Type: on Rhinolophus sp., Cantor, Sitio Tegato, Davao City, Mindanao, Philippines; MAA (1962): 435; MAA (1965): 384; DELFINADO \& HARDY (1977): 434.

Documented host records: Emballanura alecto (new host record), Eonycteris spelaea, Hipposideros ater (newhostrecord), Hipposideros galeritus, Miniopterus australis, Miniopterus schreibersi, Rhinolophus arcuatus, Rhinolophus philippinensis (new host record), Rhinolophus rufus, Rhinolophus virgo.

Material examined: on E. alecto: $\delta^{\lambda}$; on $H$. ater: $2+$; on $M$. australis: $\widehat{\partial}$,, ; on $M$. schreibersi: $2 \hat{O}$; on $R$. philippinensis: $4 \hat{O}$.

Distribution: China; Indonesia (Borneo), Malaysia, Myanmar, Philippines (Greater Luzon: Marinduque (new island record); Greater Mindanao; Greater Negros-Panay: Negros Island; Greater Palawan: mainland Palawan).

Family Nycteribiidae Samouelle, 1819

Subfamily Cyclopodiinae Maa, 1965

Genus Eucampsipoda Kolenati, 1857

Eucampsipoda KolEnATI (1857): 62. Type species: Nycteribia hyrtlii Kolenati (1856), by subsequent designation of THEODOR (1955): 196; THEODOR (1955): 196; MAA (1962): 426; MAA (1965): 380; DELFINADO \& HARDY (1977): 420.

\section{Eucampsipoda inermis Theodor, 1955}

Eucampsipoda inermis THEODOR (1955): 215. Types: on Eonycteris spelea glandifera and Rousettus amplexicaudatus, Soribao, Borongan, Samar, Philippines, in Natural History Museum (London); THEODOR (1963): 180; MAA, (1962): 426; MAA (1965): 379; DeLFINADO \& HARDY (1977): 420.

Documented host records: Eonycteris spelaea, Rousettus amplexicaudatus.

Material examined: on $R$. amplexicaudatus: $2+$.

Distribution: Australasian: Northeastern New Guinea; Oriental: Indonesia (Java, Sumba), Malaysia, Myanmar, Philippines (Greater Luzon: Catanduanes; Marinduque Island (new island record); Mindoro; Polillo Islands; Greater Mindanao: Leyte, mainland Mindanao, Samal Island, Samar; Greater Negros-Panay: Cebu, Negros; Greater Palawan: Balabac Island, Busuanga Island, mainland Palawan; Greater Sulu: Jolo.

\section{Eucampsipoda philippinensis Ferris, 1924}

Eucampsipoda philippinensis FerRIS (1924): 76. Type: female, on undetermined bat, Montalban, Rizal, Luzon, Philippines; in National Museum of Natural History (Washington, DC).; THEODOR (1955): 210; THEODOR 91963): 178; MAA (1965): 379; DELFINADO \& HARDY (1977): 421.

Documented host records: Eonycteris robusta, Hipposideros diadema, Miniopterus schreibersi, Rousettus amplexicaudatus. 
Material examined: on M. schreibersi: $4 \hat{\circ}$, + ; on $R$. amplexicaudatus: $2 \hat{\jmath}, 2+$.

Distribution: Philippines (Greater Luzon: Marinduque (new island record), Mindoro, Polillo Islands, Rizal; Greater Mindanao: Leyte, mainland Mindanao; Greater Negros-Panay: Negros Island.

\section{Genus Nycteribia Latreille, 1796}

Nycteribia LATREILle (1796): 76. Type species: Nycteribia pedicularia Latreille 1805, by designation under the Plenary powers of ICZN, Opinion 128: 493; SPEISER (1901): 11; MAA (1962): 417; MAA (1965): 381; DELFINADO \& HARDY (1977): 427.

Listripoda KolEnATI (1857): 62. Type species: Listripoda blassi Kolenati 1856, by subsequent designation of COQUILLET (1910): 562.

Acrocholidia Kolenati (1857): 62. Type species: Acrocholidia bechsteini Kolenati 1857, by subsequent designation of CoQUILLET (1910): 502.

Nycteriphila Grulich \& Povolny (1955): 116-128. Type species: Nycteribia schimidlii Schiner 1853, by original designation.

\section{Nycteribia allotopa Speiser, 1901}

Nycteribia allotopa SPEISER (1901): 47. Type: host unknown, Cave at Lian si Paghe, West Sumatra, in Natural History Museum Giacomo Doria (Genoa); FERRIS (1924): 397; THEODOR (1963): 152; MAA (1965): 382; DELFINADO \& HARDY (1977): 427.

Nycteribia insolita ScOTT (1908): 364. Type: male, on Miniopterus schreibersii, Tainan, Formosa, in Natural History Museum (London).

Listropodia wui Hsu (1935): 295. Type: male, host unknown, Soochow, China, repository unknown.

Documented host records: Megaderma spasma (new host record), Miniopterus australis, Miniopterus schreibersi, Pipistrellus imbricatus, Rhinolophus arcuatus (new host record), Rhinolophus philippinensis (new host record); Rhinolophus virgo (new host record), Tadarida luzonus.

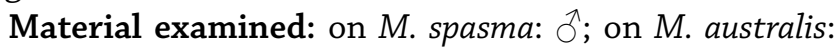
$16{ }^{\lambda}$,, ; on M. schreibersi: $10{ }^{\lambda}, 5 \bigcirc$; on R. arcuatus: $\delta^{\lambda}$; on $R$. philippinensis: ${ }^{\widehat{\partial}}$; on $R$. virgo: $\partial^{\circ}$.

Distribution: India, Indonesia (Java, Sumatra), Philippines (Greater Luzon: Marinduque (new island record), Polillo Islands; Greater Palawan).

\section{Nycteribia allotopoides Theodor, 1963}

Nycteribia allotopoides THEODOR (1963): 157. Type: male, ex Miniopterus sp., Luangbay Cave, Sitio Tegato, Davao City, Mindanao, Philippines, in Field Museum of Natural History (Chicago); MAA (1965): 382; DELFINADO \& HARDY (1977): 427.

Documented host record: Megaderma spasma (new host record), Miniopterus australis, Miniopterus schreibersi, Myotis macrotarsus, Rhinolophus philippinensis (new host record).

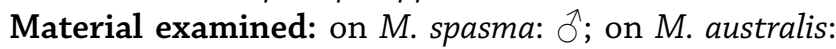

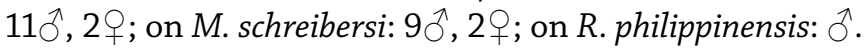

Distribution: Myanmar, Philippines (Greater Luzon: Marinduque Island (new island record), Polillo Islands; Greater Mindanao).

\section{Nycteribia parvula Speiser, 1901}

Nycteribia parvula SPEISER (1901): 48. Type: host unknown, Cave at Lian si Paghe, West Sumatra, in Natural History Museum Giacomo Doria (Genoa); FERRIS (1924): 399; THEODOR (1963): 158; MAA (1965): 382; DELFINADO \& HARDY (1977): 427.
Documented host record: Chaerephon plicata, Miniopterus australis, Miniopterus schreibersi, Rhinolophus arcuatus.

Material examined: on $M$. australis: $7 \hat{O}, 5+$; on $M$. schreibersi: $12 \hat{\jmath}, 7$.

Distribution: Australasian: New Guinea; Oriental: Afghanistan, India, Indonesia (Amboina, Java, Moluccas, Sumatera), Myanmar, Pakistan, Philippines (Greater Luzon: Marinduque (new island record), mainland Luzon, Polillo Islands; Greater Mindanao: mainland Mindanao, Tablas); Palaeotropic: Japan.

Nycteribia parvuloides Theodor, 1963

Nycteribia parvuloides THEODOR (1963): 162. Type: male, ex Miniopterus sp., Luangbay Cave, Sitio Tegato, Davao City, Mindanao, Philippines, in Field Museum of Natural History (Chicago); MAA (1965): 382; DELFINADO \& HARDY (1977): 427.

Documented host record: Eonycteris spelaea, Hipposideros coronatus, Miniopterus australis, M. schreibersi, Rhinolophus arcuatus (new host record), Rhinolophus virgo (new host record), Rousettus amplexicaudatus.

Material examined: on $M$. australis: $5 \hat{\jmath}, 3$; on $M$. schreibersi: $7 \hat{\partial}, 3$; ; on $R$. arcuatus: $\delta^{\lambda}$; on $R$. virgo: $\delta^{\lambda}$.

Distribution: Australasian: Nicobar Island; Oriental: Malaysia, Myanmar, Philippines (Greater Luzon: Marinduque Island (new island record), Polillo Islands; Greater Mindanao).

Genus Penicillidia Kolenati, 1863

Megistropoda Kolenati (1857): 62

Penicillidia Kolenati (1863): 69. Type species: Nycteribia dufourii Westwood 1834, by subsequent designation of SPEISER (1901): 32; MAA (1962): 422; MAA (1965): 379; DeLfinAdo \& HARDY (1977): 428.

\section{Penicillidia acuminata Theodor, 1963}

Penicilldia oceanica acuminata THEODOR (1963): 174. Type: ex Rhinolophus and Miniopterus spp., Miatan Cave, Katipunan, Zamboanga, Mindanao, Philippines; in Field Museum of Natural History (Chicago).

Penicillidia acuminata: MAA (1965): 374; DelfinAdo \& HARdy (1977): 428.

Documented host record: Chaerephon plicata, Emballanura alecto, Miniopterus australis, Miniopterus schreibersi.

Material examined: on M. australis: $2 \hat{\jmath}$, 2甲; on M. schreibersi: $8{ }^{\lambda}, 49$.

Distribution: Oriental: Indonesia (Java), Philippines (Greater Luzon: Lubang, Marinduque (new island record), Polillo Island; Greater Mindanao).

Penicillidia oligacantha Theodor, 1963

Penicillidia oligacantha THEODOR (1963): 171. Type: male, on Miniopterus sp., Luangbay Cave, Sitio Tegato, Davao City, Mindanao, Philippines, in Field Museum of Natural History (Chicago); MAA (1965): 379; DELFINADO \& HARDY (1977): 428.

Documented host record: Eonycteris spelaea, Miniopterus australis, Miniopterus schreibersi, Rousettus amplexicaudatus.

Material examined: on M. schreibersi: $3 \hat{\jmath}$,, .

Distribution: Oriental: Philippines (Greater Luzon: Marinduque Island (new island record), Polillo Island; Greater Mindanao). 
Genus Phthiridium Hermann, 1804

Phthiridium Hermann (1904): 12. Type species: Phthiridium biarticulatum Hermann 1804, by subsquent designation of COQUILLET (1910): 590; MAA (1965): 381; DELFINADO \& HARDY (1977): 429.

Phthiridium brachyacantha Theodor, 1963

Stylidia brachyacantha THEODOR (1963): 164. Type: male, host, unknown, Caburan, Davao, Mindanao, Philippines, in Field Museum of Natural History (Chicago).

Phthiridium brachyacantha MAA (1965): 381; DelFINADO \& HARDY (1977): 429.

Host record: Known only from Rhinolophus spp.

Material examined: On Rhinolophus arcuatus: $10^{\lambda}$.

Distribution: Oriental: Philippines (Greater Luzon: Marinduque, (new island record); Greater Mindanao).

Order Siphonaptera

Family Ischnopsyllidae Wahlgren, 1907

Genus Thaumapsylla Rothschild, 1907

Thaumapsylla RoTHSCHILD (1907): 329. Type species: Thaumapsylla breviceps Rothschild, 1907.

\section{Thaumapsylla breviceps Rothschild, 1907}

Thaumapsylla breviceps ROTHSCHILD (1907): 329.

Documented host record: Known only to infest fruitbats (Pteropodidae)

Material examined: Rousettus amplexicaudatus: $3 \hat{\jmath}, 4$ ㅇ.

Distribution: Ethiopian region (Afrotropical); Oriental: Philippines (Greater Luzon: mainland Luzon, Marinduque (new island record), Mindoro).

\section{Thaumapsylla longiforceps Traub, 1951}

Thaumapsylla longiforceps TRAUB (1951): 15.

Documented host record: Known only to infest fruitbats (Pteropodidae).

Material examined: Rousettus amplexicaudatus: $\delta \hat{\sigma}, 29$.

Distribution: Oriental: Philippines (Greater Luzon: Laguna, Marinduque (new island record); Greater Mindanao: Davao, Leyte).

Class Arachnida

Subclass Acari

Order Ixodida

Family Argasidae Koch, 1844

Genus Carios Latreille, 1796

Carios LATREILLE (1796): 176

Carios batuensis Hirst, 1929

Carios batuensis HIRst (1929): 365.

Documented host record: Known to be ectoparasitic on Pteropodidae.

Material examined: Rousettus amplexicaudatus: $\hat{O}, 2 q$.

Distribution: Oriental: Philippines (Marinduque (NCR and new island record), Indonesia (Sulawesi).

Family Spinturnicidae Oudemans, 1902
Genus Ancystropus Kolenati, 1856

Ancystropus Kolenati (1856): 1; RUdNICK (1960): 171.

Ancystropus taprobanius Turk, 1950

Meristaspis taprobanius TURK (1950): 73. Type: female, on Rousettus seminudus (Gray), Rattota, Matale District, Ceylon, in G. Thompson \& F.A. Turk collection.

Ancystropus taprobanius: RUDNICK (1960): 177.

Ancystropus indicus HiREgAUdER \& BAL (1955): 221 (nom. nud.). Type: ex Rousettus leschenaulti, Jogeshwari and Kanheri Caves, Bombay, in Hiregauder \& Bal collection, Institute of Science, Mumbai. DomRow 1972).

Ancystropus rudnicki BAKER \& DELFINADo (1964): 577. Type: female, on unknown bat, Sum-Sum, New Guinea, in Bernice Pauahi Bishop Museum (Honolulu). Domrow 1972).

Documented host record: Cynopterus brachyotis and Rousettus amplexicaudatus.

Material examined: on Hipposideros ater: $q$; on Miniopterus schreibersi: $\bigcirc$; on Rhinolophus arcuatus: $\overbrace{}^{\top}$; on Rousettus amplexicaudatus: $2 \hat{\jmath}, 2+$.

Distribution: Australasian: Papua New Guinea; Oriental: India, Laos, Philippines (Greater Luzon: Laguna, Marinduque Island (new island record); Greater NegrosPanay: Cebu, Negros Oriental), Sri Lanka.

Genus Meristaspis Kolenati, 1857

Meristaspis Kolenati (1857): 60; RUdNICK (1960): 178.

\section{Meristaspis sp. 1}

Documented host record: The genus is known to infest members of the family Pteropodidae.

Material examined: Rousettus amplexicaudatus: $\rightarrow$.

Distribution: Oriental: Philippines (Marinduque Island [new island record]).

\section{DISCUSSION}

This paper presents the first faunal survey of bat ectoparasites in Marinduque Island, and therefore, all taxa documented herein are new island records. Among the recorded families, Streblidae, Nycteribiidae, Ischnopsyllidae, and Spinturnicidae (DELFINADO \& BAKER 1963; DITTMAR et al. 2006; KRASNOV 2008) are restricted only to bats, whereas the Argasidae tend to have a much broader host specificity in various terrestrial vertebrate groups (ESTRADA-PEÑA et al. 2010; BARROs-BATTESTI et al. 2015).

The Ischnopsyllidae is the only siphonapteran family exclusively associated with bats (SMIT 1957; KRASNOV 2008). Bat fleas are traditionally divided into two subfamilies: Thaumapsyllinae (parasitic to Megachiorptera), and Ischnopsyllinae (parasitic to microchipterans) (WHITING et al. 2008). Of the three bat flea genera known to occur in the Philippines, only Thaumapsylla (Figure 2) was documented in Marinduque and was known to be associated with pteropodids.

Nycteribiidae are apterous batflies with arachnoid habitus and is most specious in the Old World (HuTson 1984). Currently, there are three recognized subfamilies known in literature, but only two, Cyclopodiinae and Nycteribiinae, 

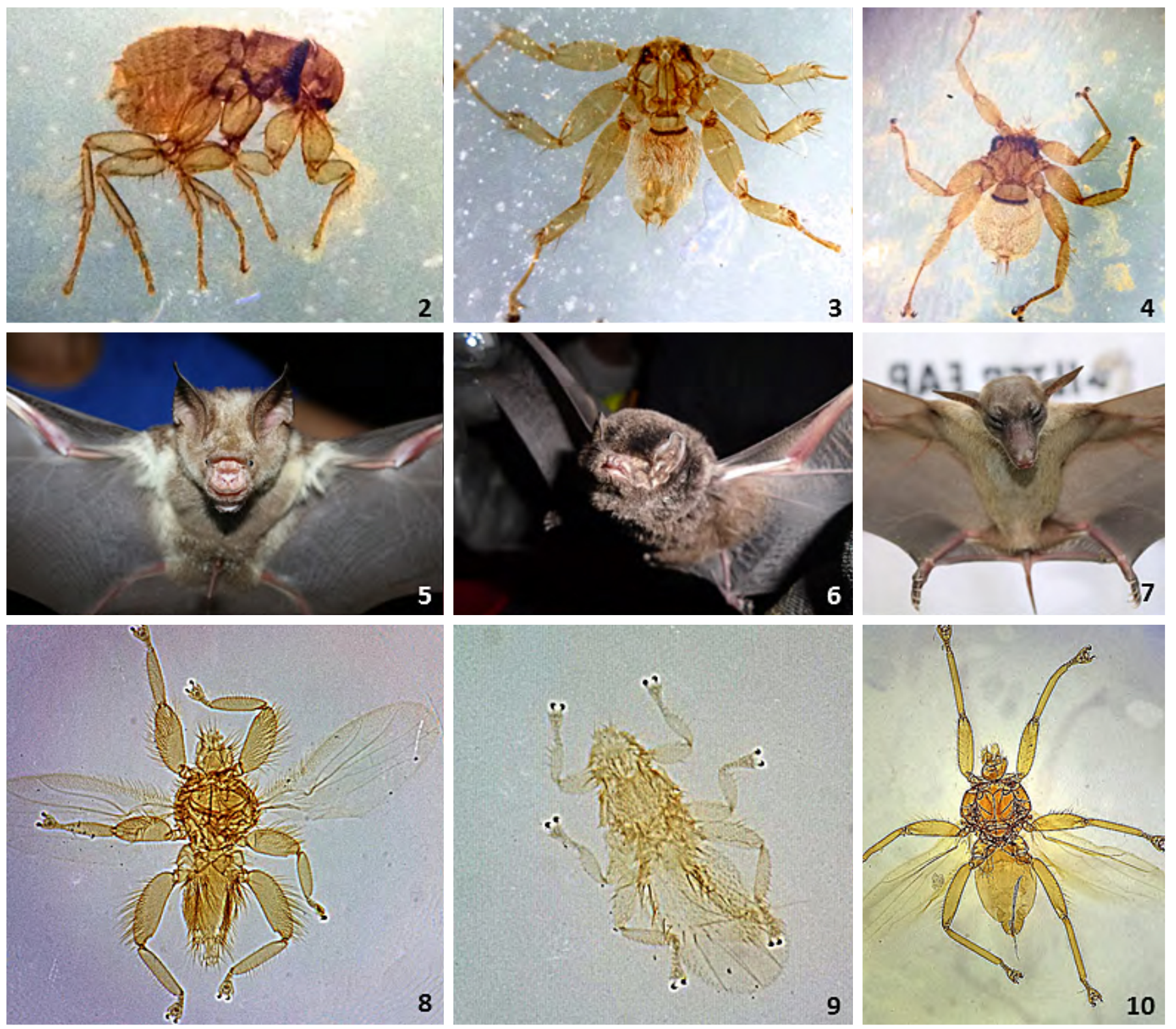

Figures 3-9. Some ectoparasitic arthropods, and bat hosts, from Marinduque Island, Philippines. 2. Thaumapsylla breviceps Rothschild, bat flea found in fruit-eating bats. 3. Nycteribiid bat fly Eucampsipoda sundaica. 4. Nycteribiid bat fly Nycteribia parvula. 5. Bat host Hipposideros diadema. 6. Bat host Miniopterus schreibersii. 7. Bat host Rousettus amplexicaudatus. 8. Streblid batfly, Brachytarsina amboinensis. 9. Streblid batfly, Raymondia pseudopagodarum. 10. Streblid batfly, Megastrebla parvior.

occur in the Philippines (MAA 1962; THEODOR 1963; CUY 1980b). In this paper, four genera were recorded: Eucampsipoda (Figure 3), Nycteribia (Figure 4), Penicillidia, and Phthiridium. Eucampsipoda is exclusively associated with Pteropodidae (MAA 1962) whereas the latter three genera are known to be associated with microchiropterans (THEODOR 1963).

The family Streblidae is usually found on insect-eating bats (Figure 5-6) and is only infrequently found on fruiteating bats (Figure 7) (MAA 1971), which was corroborated here. The genera Brachytarsina (as documented on Hipposideridae, Megadermatidae, Rhinolophidae, Vespertilionidae) (Figure 8) and Raymondia (as documented on Emballanura alecto) (Figure 9) were found in bat families belonging to the suborder Microchiroptera. The genus Megastrebla (Figure 10) is entirely ectoparasitic on cave- dwelling Megachiroptera, including Dobsonia, Eonycteris, and Rousettus (MAA 1971). In this study, Megastrebla was recorded only in Rousettus amplexicaudatus. The streblid fauna is predominantly Indo-Malayan with endemicity of 50\% at the species level (AMARGA et al. 2015).

Spinturnicidae (Acarina) comprises mites that are exclusively ectoparasitic on bats. Four genera occur in the Philippines and two of them, Ancystropus and Meristaspis, are documented in Marinduque Island. In the Philippines, the genus Ancystropus is known only from pteropodids (CUY 1979; Delfinado \& BAKER 1963). New host records for Ancystropus taprobanius include microchiropterans ( $R h i-$ nolophus, Hipposideros, and Miniopterus). The genus Meristaspis was documented on the family Pteropodidae (CUY 1979), and in Marinduque, it was collected from Rousettus amplexicaudatus. 
New host records in the Philippines for Brachytarsina cucullata (on Hipposideros diadema griseus, Megaderma spasma, Miniopterus australis, Rhinolophus philippinensis, and Rhinolophus arcuatus), Brachytarsina proxima (on Miniopterus schreibersi and Meg. spasma), Brachytarsina werneri (on $M$. australis, M. schreibersi, Meg. spasma, and R. arcuatus), Raymondia pseudopagodarum (on R. philippinensis, Hipposideros ater, and Emballanura alecto), Eucampsipoda philippinensis (on M. schreibersi), Nycteribia allotopa (on Rhinolophus sp. and Meg. spasma), Nycteribia allotopoides (on Meg. spasma and $R$. philippinensis), Nycteribia parvuloides ( $R$. arcuatus and $R$. virgo), Ancystropus taprobanius (on R. arcuatus, $H$. ater, and M. schreibersi) and Carios batuensis (on Rousettus amplexicaudatus).

This is the first study of the first faunal surveys in the Philippines which addresses only the ectoparasite diversity of cave-dwelling chiropterans. Previous studies of the bat ectoparasite fauna of Philippine bats also included noncave dwelling bats (THEODOR 1963; DELFINADO \& BAKER 1963; CUy 1979, 1980a, 1980b; HASTRITER \& BUSH 2013).

Ectoparasites are integral components of the ecosystem and to some extent may portray the role of keystone species. They have evolved in parallel with their host taxa over time. Their variety of host specificity spectrum ranging from monoxeny to polyxeny can also be used to elucidate their evolutionary radiation within the host taxa. Bat ectoparasites are important groups that should be studied in documenting Philippine biodiversity.

\section{ACKNOWLEDGEMENTS}

We gratefully acknowledge the support of the following persons and institutions: University of the Philippines Los Baños Basic Research Program, University of the Philippines Los Baños Museum of Natural History, Local Government Unit of Marinduque Island, Mr. Edison Cosico, Mr. Eduardo Eres, Mr. Joseph Paolo Macasaet, Mr. Russel Baniqued, Dr. Juan Carlos Gonzalez, Dr. Leonila Corpuz- Raros, Prof. Mateo Zipagan, Dr. Bruce Patterson, and Dr. Leticia Espiritu- Afuang.

\section{LITERATURE CITED}

Alvarez, J.D.V., Lit, JR., I.L. \& Alviola, P.A. 2015. Bat flies (Diptera Nycteribiidae) from Mount Makiling, Luzon Island: new host and distribution records, with a checklist of species found in the Philippines. Check List 11(1): 1509. doi: 10.15560/15.1.1509

AMARGA, A.K., YAP, S. \& ADORADA, J.R. 2015. Biogeographic distribution of Streblidae in the Philippines (Diptera: Brachycera: Calyptratae). Transactions of the National Academy of Science and Technology 37(1): 75.

BaKer, E.W. \& Delfinado, M.D. 1964. Spinturnicidae of the South East Asia and the Pacific region. Pacific Insects 6(4): 571-591. http://hbs.bishopmuseum.org/pi/pdf/6(4)-571.pdf

Barros-Battesti, D.M., LANdulfo, G.A., Luz, H.R., Marcili, A., OnOFRIO, V.C. \& FAMADAS, K.M. 2015. Ornithodoros faccinii n. sp. (Acari: Ixodida: Argasidae) parasitizing the frog Thoropa miliaris (Amphibia: Anura: Cycloramphidae) in Brazil. Parasites and Vectors 8: 268. doi: 10.1186/s13071-015-0877-3

BochKov, A.V., KLOMPEN, H. \& OCONNOR, B.M. 2008. Review of external morphology of Chirorhynchobiidae (Acari: Sarcoptoidea) with description of a new species. Journal of Medical Entomology 45(2): 193-202. doi: http://doi.org/dnxjkg

Coquillet, D.W. 1910. The type species of North American genera of Diptera. Proceedings of United States National Museum 37: 499-647

CuY, L.S. 1980a. Nycteriboscinae (Diptera: Streblidae) of the Philippines. Philippine Journal of Science 9(2-3): 137-144.

CuY, L.S. 1980b. Nycteribiidae (Diptera) of the Philippines. Philippine Journal of Science 9(2-3): 145-168.

CuY, L.S. 1979. Synopsis of Philippine Spinturnicidae (Acarina: Mesostigmata). Kalikasan, Philippine Journal of Biology 8(2): 162-172.

Delfinado, M.D. \& BAKer, E.W. 1963. Mites of the family Spinturnicidae from the Philippines (Acarina). Pacific Insects 5(4): 905-920. http://hbs.bishopmuseum.org/pi/pdf/5(4)-905.pdf

DELFINADO, M.D. \& HARDY, D.E. 1975. A catalog of the Diptera of the Oriental Region. Vol. II: Suborder Brachycera through Aschiza, Suborder Cyclorrhapha. Honolulu: University Press of Hawaii 459 pp.

DelfinAdo, M.D. \& HARDY, D.E. 1977. A catalog of the Diptera of the Oriental Region. Vol.III: Suborder Cyclorrhapha (excluding division Aschiza). Honolulu: University Press of Hawaii 854 pp.

Dittmar, K., Porter, M.L., Murray, S. \& Whiting, M.F. 2006. Molecular phylogenetic analysis of nycteribiid and streblid bat flies (Diptera: Brachycera, Calyptratae): implications for host associations and phylogeographic origins. Molecular Phylogenetics and Evolution 38: 155-170. doi: 10.1016/j.ympev.2005.06.008

Domrow, R. 1972. Acari Spinturnicidae from Australia and New Guinea. Acarologia 13: 552-584.

Durden, L.A., Best, T.L, Wilson, N. \& Hilton, D.C. 1992. Ectoparasitic mites (Acari) of sympatric Brazilian free-tailed bats and big brown bats in Alabama. Journal of Medical Entomology 29: 507-511.

Durden, L.A., Merker, S., Beati, L. 2008. The tick fauna of Sulawesi, Indonesia (Acari: Ixodoidea: Argasidae and Ixodidae). Experimental and Applied Acarology 45(1-2): 85-110. doi: 10.1007/ s10493-008-9144-z

EstradA-PeÑA, A. \& LA CRUZ, J. DE. 1992. Ewingana (Doreyana) baeklandae, sp. n., a parasite of molossid bats in Spain (Acari, Myobiidae). Folia Parasitologica 39: 67-73.

Estrada-Peña, A., Mangold, A.J., NAVA, S., VenZal, J.M., Labruna, M. \& Guglielmone, A.A. 2010. A review of the systematics of the tick family Argasidae (Ixodida). Acarologia 50(3): 317-333. doi: 10.1051/acarologia/20101975

FAIN, A. 1979. Diagnoses de nouveaux Labidocarpinae parasites de chiroptères asiatiques (Acari, Astigmata). Bulletin et Annales de la Société Royale d'Entomologie de Belgique 115: 264-266.

FAIN, A. 1980. The labidocarpine mites (Acarina: Chirodiscidae) from Oriental bats. I. Genera Afrolabidocarpus Fain, 1970 and Asiolabidocarpus Fain, 1972. International Journal of Acarology 6: 131-140.

FAIN, A. 2002. Notes on a small collection of mites (Acari) parasitic on bats in the Philippines. Acarologia 42(1): 67-74.

FAIN, A. \& Lukoschus, F.S. 1971. Parasitic mites of Surinam. 15. Nasal ereynetid mites of bats with a key to the known species (Trombidiformes). Bulletin et Annales de la Société Royal Belgique (Entomologie) 107: 284-297.

Ferris, G.F. 1924. Two Diptera Pupipara from Philippine bats. Philippine Journal of Science 24: 73-79.

FrAUENFELD, G.R. 1855. Über eine neue Fliegengattung Raymondia aus der Familie Coriaceen nebst Beschreibung zweier Arten derselben. Kaiserliche Akademie der Wissenschaften zu Wien. Sitzungsberichte der Mathematisch-naturwissenschaftlichen Klasse 18: 320-333. http://biodiversitylibrary.org/page/6441889 Frauenfeld, G.R. 1856. Über Raymondia Fr., Strebla Wd. und 
Brachytarsina Maq. Kaiserliche Akademie der Wissenschaften zu Wien. Sitzungsberichte der Mathematisch-naturwissenschaftlichen Klasse 18(22): 468-478. http://biodiversitylibrary.org/ page/6364151

Grulich, I. \& Povolny, D. 1955. Faunistich-Bionomische Uebersicht der Nycteribiidae aus Gebiete der CRS. Zoologiscke a Entomologicke Listy 4: 111-134.

HASTRITER, M.W. \& BUSH, S.E. 2013. Description of Lentistivalius philippinensis, a new species of flea (Siphonaptera, Pygiosyllomorpha, Stivaliidae), and new records of Ascodipterinae (Streblidae) on bats and other small mammals from Luzon, The Philippines. Zookeys 260: 17-30. doi: 10.3897/zookeys.260.3971

Heddergott, M. 2008. Two new species of Parichoronyssus Radovsky, 1966 (Acari, Dermanyssoidea, Macronyssidae) from bats of the genus Phyllostomus (Mammalia, Chiroptera, Phyllostomidae) in Paraguay and Cuba. Spixiana 31(2): 183-193.

HERmanN, J.F. 1804. Memoire apterologique. Strasbourg: F.G. Levrault. 144 pp.

HIREgAUDAR, L.S. \& BAL, D.V. 1955. Ectoparasites of some bats from India. Current Science 24(7): 221-222.

HIRst, S. 1929. Fauna of the Batu Caves, Selangor. XI. Arachnida: Acarina (Ixodidae). Journal of Federated States Museum 14(3-4): 365.

HoRvath, G. 1910. Description of a new bat-bug from British Columbia. Entomologist's Monthly Magazine (series 2) 21:12-13. http://biodiversitylibrary.org/page/9270611

Hsu, Y.C. 1935. Two new species of insect parasites of the bat in Soochow. Peking Natural History Bulletin 9: 293-298.

Hutson, A.M. 1984. Handbook for the identification of British Insects. Diptera, Hippoboscidae and Nycteribiidae. Royal Entomological Society of London $39 \mathrm{pp}$.

JenYnS, L. 1839. On three undescribed species of the genus Cimex, closely allied to the common bed-bug. The Annals of Natural History; or Magazine of Zoology, Botany, and Geology 3: 241-244. http://biodiversitylibrary.org/page/3387840

Jobling, B. 1930. A revision of the genus Raymondia Frauenfeld. Parasitology 22(3): 283-301. doi: 10.1017/S003118200001115X

Jobling, B. 1934. A revision of the genus Nycteribosca Speiser (Diptera, Pupipara, Streblidae). Parasitology 26(1): 64-97. doi: 10.1017/S0031182000023337

Jobling, B. 1951. A record of the Streblidae from the Philippines and other Pacific islands, including morphology of the abdomen, host-parasite relationship and geographic distribution, and with description of five new species (Diptera). Transactions of the Entomological Society of London 102: 211-246.

JORDAN, K. \& N.C. RoTHSCHILD. 1921. New genera and species of bat fleas. Ectoparasites 1: 142-162.

KLOMPEN, J.S.H. \& OCONNOR, B.M. 1987. The systematic position of the genus Chirobia Fain, 1959 (Astigmata: Sarcoptidae) with a description of Chirobia harpyionycteris $\mathrm{n}$. sp. from the Old World fruit bat Harpyionycteris whiteheadi (Chiroptera: Pteropodidae). Systematic Parasitology 9(2): 153-161. doi: 10.1007/BF00012193

KocH, C.L. 1844. Systematische Uebersicht über die Ordnung der Zecken. Archive für Naturgeschichte 10(1): 217-239. http://biodiversitylibrary.org/page/9697358

Kolenati, F.A. 1856. Die parasite der Chiropteren. Brünn: Rohrer. $51 \mathrm{pp}$.

KolENATI, F.A. 1857. Synopsis prodromal der Nycteribien. Wiener Entomologishe Monatsschrift 1: 61-62.

KolenAti, F.A. 1863. Beitrag zur Kenntnis der Phthirio-Myiarien. Horae Societatis Entomologicae Rossicae 2: 9-109. http://biodiversitylibrary.org/page/47976301

KRASNOV, B. 2008. Functional and evolutionary ecology of fleas. A model for ecological parasitology. Cambridge: Cambridge University Press 583 pp.

LATREILlE, P.A. 1796. Précis des caractères génériques des insectes, disposés dans un ordre naturel. Brive: F. Bordeaux. 202 pp. doi: 10.5962/bhl.title.58411

LATREILLE, P.A. 1805. Histoire naturelle, generale et particulare des crustaces et des Insectes 14: 1-432.

MAA, T.C. 1962. Records and descriptions of Nycteribiidae and Streblidae (Diptera). Pacific Insects 4(2): 417-436. http://hbs. bishopmuseum.org/pi/pdf/4(2)-417.pdf

MAA, T.C. 1965. An interim world list of bat flies. Journal of Medical Entomology 1: 377-386.

MAA, T.C. 1971. Review of the Streblidae (Diptera) parasitic on megachiropteran bats. Pacific Insects Monograph 28: 213-243. http://hbs.bishopmuseum.org/pim/pdf/pim28-213.pdf

MACQUART, P.J.M. 1851. Diptères exotiques nouveaux ou peu connus. Suite du $4^{\mathrm{e}}$ supplement. Mémoirs de la Société des Sciences, de l'Agriculture et des Arts, de Lille 1850: 134-294. http://biodiversitylibrary.org/page/34651081

MARShALL, A.G. 1982. Ecology of insects ectoparasitic on bats; pp. 369-401, in: KunZ, T.H. Ecology of bats. New York: Plenum Press.

NAKATA, S. \& MAA, T.C. 1974. A review of the parasitic earwigs. Pacific Insects 16(2-3): 307-374. http://hbs.bishopmuseum.org/ pi/pdf/16(2)-307.pdf

OUDEMANS, A.C. 1902. Acarologische aantekeningen. Entomologische Berichten 1: 43-45. http://biodiversitylibrary.org/page/8982420

RonDANI, C. 1878. Muscaria exotica Musei civici Januensis observata et distincta. Fragmentum IV. Hippoboscita exotica non vel minus cognita. Annali del Museo civico di storia natural di Genova 12: 150-169. http://biodiversitylibrary.org/page/30263718

Rothschild, M. 1907. Some new Siphonaptera. Novitates Zoologicae 14: 329-333. http://biodiversitylibrary.org/page/2989501

RUDNICK, A. 1960. A revision of the mites of the family Spinturnicidae. University of California Publications in Entomology 17: 157-283.

SAMOUELLE, G. 1819. The Entomologist's useful compendium or an introduction to the knowledge of British insects. London: Thomas Boys. 496 pp. doi: 10.5962/bhl.title.34177

SCHINER, J. 1853. Dipterologische Fragmente II. Verhandlungen des Zoologisch-Botanischen Vereins in Wien 3: 150-154. http:// biodiversitylibrary.org/page/26711365

ScotT, H. 1908. On certain Nycteribiidae, with description of two new species from Formosa. Transactions of the Entomological Society of London 1908: 359-370. http://biodiversitylibrary.org/ page/14685868

SHAW, M.D. 2011. Laelapidae (Acari: Mesostigmata) on megachiropteran bats: new records and a new species of Neolaelaps Hirst. Zootaxa 2807: 41-56.

SMIT, F.G.A.M. 1957. Handbooks for the identification of British insects. Siphonaptera. Royal Entomological Society of London $94 \mathrm{pp}$.

SPEISER, P.G.E. 1900. Ueber die Strebliden, Fledermausparasiten aus der Gruppe der pupiparen Dipteren. Archiv für Naturgeschichte 66: 31-70.

SPEISER, P.G.E. 1901. Uber die Nycteribien, Fledermausparasiten aus Gruppe der pupiparen Dipteren. Archiv für Naturgeschichte 67: 11-78.

THEODOR, O. 1955. On the genus Eucampsipoda Kolenati and Dipseliopoda n. g. (Nycteribiidae, Diptera). Parasitology 45: 195-229.

Theodor, O. 1963. Philippine batflies of the family Nycteribiidae (Diptera, Pupipara). Fieldiana (Zoology) 42(11): 151-192. http:// biodiversitylibrary.org/page/2836554

Tipton, V. \& Boese, J.L. 1958. Steatonyssus furmani, a new Nearctic bat mite. Proceedings of the Entomological Society of Washington 60(2): 80-84. http://biodiversitylibrary.org/page/26237319

TraUb, R. 1951. Fleas collected by the Chicago Natural History Expedition to the Philippines 1946-1947. Proceedings of the Biological Society of Washington 64: 1-21. http://biodiversitylibrary.org/ page/34566961

TURK, F.A. 1950. Studies of Acari-VI. Parasitic mites from mam- 
malian hosts obtained in Ceylon. Parasitology 40(1): 63-76.

UchIKAWA, K. 1985. Mites of the genus Pteracarus (Acarina, Myobiidae) taken from the bats of the genus Miniopterus (Chiroptera, Miniopteridae). Zoological Science (Tokyo) 2: 109-114.

Wahlgren, E. 1907. Svenska Siphonaptera. Entomologisk Tidsskrift 28: 85-91.

Westwood, J.C. 1835. On Nycteribia, a genus of wingless insects. Transactions of the Zoological Society of London 1: 275-294. doi: 10.1111/j.1096-3642.1835.tb00626.x

Whiting, M.F., Whiting, A.S., Hastriter, M.W. \& DitTMar, K. 2008. A molecular phylogeny of fleas (Insecta: Siphonaptera): origins and host associations. Cladistics 24: 1-31. doi: 10.1111/ j.1096-0031.2008.00211.x

ZABAT, A.G. \& EDUARDO, S.L. 2011. Some ectoparasites of the Com- mon Rousette Bat (Rousettus amplexicaudatus Geofroy, 1810) (Mammalia: Chiroptera: Pteropodidae) from colonies in Batangas and Rizal, Philippines. Philippine Journal of Veterinary Medicine 48(1): 53-56.

Authors' contributions: AKSA wrote the text, collected the data, and identified the batflies and bat hosts; PAA collected the data and identified the bat hosts; ILLJr edited the text; SAY made the figures and edited the text.

Received: 6 October 2015

Accepted: 4 September 2016

Academic editor: Gustavo Silva de Miranda 